\title{
Un estudio exploratorio sobre el estudio de implementación de procesos de gestión del conocimiento en organizaciones desarrolladoras de software en Colombia
}

FECHA DE RECEPCIÓN: 17 de enero FECHA DE APROBACIÓN: 1 de febrero Pp. 73-90

\section{Resumen}

En este artículo se presenta un estudio exploratorio sobre el estado de implementación y la importancia de ocho procesos de Gestión del Conocimiento (GC) en 169 Organizaciones Desarrolladoras de software (ODS) en Colombia. Los datos se recopilaron en una encuesta, en la que se indagó por el estado de la implementación de ocho procesos de GC propuestos en el Modelo de Referencia de Procesos de Gestión del Conocimiento (MRPGC), desde la perspectiva del logro de los resultados esperados y sus propósitos. Los resultados muestran que en las ODS en Colombia se reconoce la importancia de la GC, pero el estado de implementación de los procesos es apenas aceptable.

\section{Palabras clave}

Procesos de gestión de conocimiento, organizaciones desarrolladoras de software, Colombia, estudio exploratorio.

\section{Ernesto Amaru Galvis-Lista}

Doctor en Ingeniería de Sistemas y Computación; profesor asociado Facultad de Ingeniería, Universidad del Magdalena.

\section{Mayda Patricia González-Zabala}

Doctora en Ingeniería de Sistemas y Computación de la Universidad Nacional de Colombia; profesor de planta Universidad del Magdalena.

\section{Jenny Marcela Sánchez-Torres}

Doctora en Economía y Gestión de la Innovación y Política Tecnológica; programa Interuniversitario de las Universidades Complutense, Politécnica y Autónoma de Madrid. España; profesora Titular de la Universidad Nacional de Colombia. 


\title{
An Exploratory Study on the Implementation Status of Knowledge Management Processes in Software Development Organizations in Colombia
}

\begin{abstract}
Abstrac. This article describes a research study about the current state of the implementation and importance of eight processes of knowledge management (KM) in 169 organizations which develop software in Colombia. Data was collected from a poll used to explore the current state of the implementation of eight processes of knowledge management proposed by the Model of Reference of Knowledge Management Processes from the perspective of expected outcomes and purposes. Results show that organizations developing software in Colombia really take into account the relevance of knowledge management, but their implementation stage of processes is still acceptable.
\end{abstract}

Key words. Knowledge management processes, software development organizations, Colombia, research study.

\section{Etude exploratoire du statut de mise en place du processus de gestion des connaissances chez les fabricants de logiciels en Colombie}

Resumé. Cet article présente une étude exploratoire de la mise en place et de l'importance de huit processus de Gestion des Connaissances (GC) de 169 entreprises fabricantes de logiciel (EFL) en Colombie. Les données sont compilées à partir d'une enquête montrant l'état d'implémentation de huit processus de GC proposés à partir du Modèle de Référence des Processus de Gestion de la Connaissance (MRPGC) depuis une perspective de succès des résultats attendus et des objectifs à atteindre. Les résultats montrent qu'en Colombie les EFL attachent de l'importance à la GC en dépit de l'état de mise en place des processus qui est à peine acceptable.

Mots clefs. Processus de gestion des connaissances, entreprises fabricantes de logiciel, Colombie, étude exploratoire.

\section{Um Estudo Exploratório sobre o estado de implementação de processos de gestão de conhecimento em organizações de desenvolvimento de software na Colômbia}

Resumo. Este artigo apresenta um estudo exploratório sobre o estado de implementação e a importância de oito processos de Gestão do Conhecimento (GC) em 169 organizações de desenvolvimento de software (ODS) na Colômbia. Os dados foram recolhidos através de uma pesquisa onde se indagou sobre o estado da implementação de oito processos de GC propostos no modelo de referência de processos de Gestão do Conhecimento (MRPGC) a partir da perspectiva de alcançar os resultados esperados e seus propósitos. Os resultados mostram que nas ODS na Colômbia se reconhece a importância da GC, mas o estado de implementação dos processos é apenas aceitável.

Palabras chave. Processos de gestão do conhecimento, as organizações de desenvolvimento de software, Colômbia, estudo exploratório. 


\section{Introducción}

$\mathbf{E}$ n los últimos años se han realizado varios estudios de caracterización de las organizaciones de la industria de software en Colombia, donde se muestran los rasgos generales y el estado de avance en aspectos importantes para la industria, algunos de los cuales están relacionados con la Gestión del Conocimiento (GC) (CIDEl et al., 2013; Departamento Nacional de Planeación, 2007; Fedesoft, 2011, 2012; Fedesoft y Proexport, 2004; GRUPODEESTUDIOSSECTORIALES-DNP, 2008; McKinsey \& Company, 2008; Ministerio de Comercio, Industria y Turísmo, 2008; Ministerio de Comunicaciones, 2008; Parra Castrillón, 2008). En este tema en particular, la Federación Colombiana de la Industria del Software y Tecnologías de la Información (FEDESOFT), identificó que en las empresas del sector existe bajo grado de especialización, se desaprovechan las oportunidades identificadas en las tendencias mundiales del mercado del software por preferir el desarrollo de líneas de negocio tradicionales, y hay poca o nula dedicación a actividades de investigación, desarrollo e innovación (Fedesoft, 2012).
En este sentido, al tener en cuenta la importancia que tiene la GC en la industria del software (Bjørnson \& Dingsøyr, 2008; Dingsøyr, Bjornson, \& Shull, 2009), surge el interés por obtener evidencia empírica sobre el estado actual de la implementación de procesos de GC en las ODS del país, así como por identificar los acuerdos sobre la pertinencia e importancia de tales procesos para las ODS. Para esto, se llevó a cabo un estudio exploratorio con 169 ODS colombianas donde se tomó como referencia la versión 0.1 del Modelo de Referencia de Procesos de Gestión del Conocimiento (MRPGC) (Galvis-Lista y Sánchez-Torres, 2013). En este modelo se especifican ocho procesos de GC y sus propósitos (Tabla 1). Además, se describen 47 resultados esperados de la implementación de los procesos, los cuales pueden ser de tres tipos: producción de un artefacto, cambio significativo de estado o cumplimiento de restricciones específicas (Tabla 2). 


\section{Tabla 1. Propósitos de los procesos de GC del MRPGC v.01}

\begin{tabular}{|c|c|}
\hline Proceso & Propósito \\
\hline Identificación & $\begin{array}{l}\text { Determinar el estado actual del conocimiento organizacional y las necesidades de conocimiento } \\
\text { de la organización. Las necesidades de conocimiento de la organización pueden ser satisfechas } \\
\text { por la adquisición de conocimiento por fuera de la organización o la creación del conocimiento } \\
\text { dentro de la organización. }\end{array}$ \\
\hline Adquisición & $\begin{array}{l}\text { Adquirir conocimiento de fuentes externas a la organización. El conocimiento adquirido debería } \\
\text { satisfacer necesidades de conocimiento de la organización o debería permitir la innovación. La } \\
\text { adquisición de conocimiento implica considerar los aspectos éticos y legales del acceso y uso de } \\
\text { conocimiento de terceros. }\end{array}$ \\
\hline Creación & $\begin{array}{l}\text { Crear conocimiento organizacional. El conocimiento es nuevo cuando satisface necesidades de } \\
\text { conocimiento de la organización o cuando permite la innovación. }\end{array}$ \\
\hline Codificación & $\begin{array}{l}\text { Representar o expresar explícitamente, el conocimiento tácito de las personas en la organización } \\
\text { La codificación del conocimiento es la transformación de conocimiento tácito en conocimiento } \\
\text { explícito. La codificación incluye organización, almacenamiento y recuperación de conocimiento } \\
\text { explícito. }\end{array}$ \\
\hline Aplicación & $\begin{array}{l}\text { Utilizar el conocimiento organizacional para lograr los propósitos y objetivos organizacionales. El } \\
\text { conocimiento organizacional debería ser aplicado en situaciones como: formulación de estrategias, } \\
\text { toma de decisiones, definición y ejecución de procesos, realización de actividades, resolución de } \\
\text { problemas, o creación y evolución de productos. }\end{array}$ \\
\hline Transferencia & $\begin{array}{l}\text { Transferir conocimiento organizacional entre las personas dentro de la organización. La } \\
\text { transferencia de conocimiento implica la existencia de fuentes de conocimiento y receptores de } \\
\text { conocimiento; e involucra el aseguramiento de la asimilación del conocimiento por los receptores. }\end{array}$ \\
\hline Protección & $\begin{array}{l}\text { Proteger el conocimiento organizacional de usos ilegales o no autorizados, y explotar los usos } \\
\text { autorizados en generar beneficios para la organización. }\end{array}$ \\
\hline Evaluación & $\begin{array}{l}\text { Evaluar el conocimiento organizacional para obtener realimentación sobre su estado actual y su } \\
\text { efectividad en la organización y su entorno. }\end{array}$ \\
\hline
\end{tabular}

\section{Fuente. Galvis-Lista y Sánchez-Torres, (2013).}

\section{Tabla 2. Resultados de los procesos de GC del MRPGC v.01}

\begin{tabular}{|c|l|}
\hline \multicolumn{1}{|c|}{$\mathbf{P}$} & \multicolumn{1}{c|}{ Resultados de la implementación efectiva del proceso } \\
\hline \multirow{5}{*}{ Identificación } & $\begin{array}{l}\text { IdC1- Se construyen mapas de conocimiento para identificar y localizar el conocimiento } \\
\text { organizacional. }\end{array}$ \\
\cline { 2 - 3 } & $\begin{array}{l}\text { IdC2 - Se identifican necesidades de conocimiento de la organización. Estas necesidades pueden } \\
\text { estar asociadas a la adquisición o creación de conocimiento, o a la actualización del conocimiento } \\
\text { existente. }\end{array}$ \\
\cline { 2 - 3 } Adquisición & $\begin{array}{l}\text { IdC3 - Las necesidades de conocimiento de la organización son analizadas para determinar qué } \\
\text { tipo de conocimiento se requiere y para resolver conflictos entre las necesidades de conocimiento } \\
\text { identificadas. }\end{array}$ \\
\cline { 2 - 3 } & $\begin{array}{l}\text { IdC4 - Las necesidades de conocimiento de la organización son especificadas para describir el tipo } \\
\text { de conocimiento que se requiere y las alternativas disponibles para suplirlas. }\end{array}$ \\
\hline & $\begin{array}{l}\text { AdC1 - } \\
\text { Se identifica el conocimiento de otras organizaciones-competidores, socios, clientes o proveedores- } \\
\text { que se considera relevante para satisfacer necesidades de conocimiento de la organización. }\end{array}$ \\
\cline { 2 - 3 } & $\begin{array}{l}\text { AdC2 - Se establecen estrategias de colaboración entre la organización y sus socios de negocio } \\
\text { para la adquisición de conocimiento. }\end{array}$ \\
\hline
\end{tabular}




\section{Tabla 2. Resultados de los procesos de GC del MRPGC v.01 (Continuación)}

\begin{tabular}{|c|c|}
\hline \multirow{7}{*}{ Adquisición } & $\begin{array}{l}\text { AdC3 - Se desarrollan periódicamente reportes de vigilancia tecnológica, competitiva, comercial } \\
\text { y del entorno, que permitan identificar tendencias en el conocimiento externo para definir y } \\
\text { estructurar hojas de ruta. }\end{array}$ \\
\hline & $\begin{array}{l}\text { AdC4 - Se establecen y ejecutan procedimientos de búsqueda de talentos para contratar personas } \\
\text { con conocimientos relevantes para satisfacer necesidades de conocimiento de la organización. }\end{array}$ \\
\hline & $\begin{array}{l}\text { AdC5 - Se involucra a los miembros de la organización en programas de formación por fuera de } \\
\text { la organización. }\end{array}$ \\
\hline & $\begin{array}{l}\text { AdC6- Se adquieren activos de propiedad intelectual como patentes, marcas registradas, derechos } \\
\text { de autor o secretos industriales. }\end{array}$ \\
\hline & $\begin{array}{l}\text { AdC7 - Se adquieren herramientas o equipos cuya incorporación implique la absorción de } \\
\text { conocimiento relativo a su uso. }\end{array}$ \\
\hline & $\begin{array}{l}\text { AdC8 - Se establecen y mantienen criterios para juzgar la relevancia y accesibilidad del conocimiento } \\
\text { externo identificado como relevante. }\end{array}$ \\
\hline & AdC9 - El conocimiento externo adquirido es adaptado al entorno organizacional. \\
\hline \multirow{7}{*}{ Creación } & $\begin{array}{l}\text { CrC1 - Se identifican, registran y evalúan nuevas ideas relevantes para satisfacer las necesidades } \\
\text { de conocimiento de la organización o para tomar ventaja de las tendencias de conocimiento de } \\
\text { su entorno. }\end{array}$ \\
\hline & $\begin{array}{l}\text { CrC2 - Las ideas relevantes se desarrollan y adaptan al entorno de aplicación de la organización, } \\
\text { generando nuevo conocimiento. }\end{array}$ \\
\hline & $\begin{array}{l}\text { CrC3 - Se construyen y mantienen diferentes tipos de espacios de interacción físicos o virtuales } \\
\text { para habilitar el surgimiento de nuevas ideas. }\end{array}$ \\
\hline & $\begin{array}{l}\text { CrC4 - Se estimula a los miembros de la organización para que actúen de forma autónoma en } \\
\text { equipos auto-organizados y multifuncionales para generar ideas y desarrollar nuevos conocimientos. }\end{array}$ \\
\hline & $\begin{array}{l}\text { CrC5 - Se mantienen diferentes tipos de redundancia -información, funciones y rutinas- al interior } \\
\text { de la organización. }\end{array}$ \\
\hline & $\begin{array}{l}\text { CrC6 - Se ejecutan proyectos de investigación, desarrollo e innovación, para desarrollar las ideas } \\
\text { relevantes y convertirlas en nuevo conocimiento organizacional. }\end{array}$ \\
\hline & $\begin{array}{l}\text { CrC7 - Se entregan recompensas o estímulos a los miembros de la organización por sus } \\
\text { contribuciones en la creación de conocimiento. }\end{array}$ \\
\hline \multirow{7}{*}{ Codificación } & $\begin{array}{l}\text { CoC1 - Dependiendo del tipo de conocimiento, se seleccionan estándares y medios para su } \\
\text { representación, almacenamiento y recuperación. }\end{array}$ \\
\hline & $\begin{array}{l}\text { CoC2 - Se construyen paquetes de conocimiento codificado utilizando los estándares y medios } \\
\text { seleccionados para la representación y almacenamiento del conocimiento. }\end{array}$ \\
\hline & $\begin{array}{l}\text { CoC3 - Los paquetes de conocimiento codificado son descritos utilizando metadatos que faciliten } \\
\text { la búsqueda y recuperación. }\end{array}$ \\
\hline & $\begin{array}{l}\text { CoC4 - Los paquetes de conocimiento codificado son integrados a los diferentes medios de } \\
\text { almacenamiento o repositorios de conocimiento, utilizando varias formas de organización o } \\
\text { categorización. }\end{array}$ \\
\hline & $\begin{array}{l}\text { CoC5 -Los paquetes de conocimiento codificado están disponibles para su uso, y su existencia es } \\
\text { comunicada a las personas dentro de la organización. }\end{array}$ \\
\hline & $\begin{array}{l}\text { CoC6 - Estrategias, métodos y herramientas para recuperar el conocimiento codificado que se } \\
\text { encuentre almacenado en los diferentes medios o repositorio, está disponibles para ser usadas. }\end{array}$ \\
\hline & $\begin{array}{l}\text { CoC7 - Se ejecutan análisis periódicos sobre el uso de los paquetes de conocimiento codificado } \\
\text { para identificar aquellos que deben ser actualizados o desechados }\end{array}$ \\
\hline Aplicación & $\begin{array}{l}\text { ApC1 - El conocimiento organizacional es utilizado por las personas dentro de la organización } \\
\text { para aprender y desarrollar habilidades que permitan mejorar la forma en que se ejecutan las } \\
\text { actividades. }\end{array}$ \\
\hline
\end{tabular}




\section{Tabla 2. Resultados de los procesos de GC del MRPGC v.01 (Continuación)}

\begin{tabular}{|c|c|}
\hline \multirow{5}{*}{ Aplicación } & $\begin{array}{l}\text { ApC2 - El diseño de cargos y la asignación de los empleados a las actividades se hacen con base } \\
\text { en el conocimiento que estos poseen y que estén en disposición de aplicar en la ejecución de las } \\
\text { actividades. }\end{array}$ \\
\hline & $\begin{array}{l}\text { ApC3 - Se desarrollan nuevos productos o se mejoran los existentes, por la aplicación del } \\
\text { conocimiento organizacional. }\end{array}$ \\
\hline & ApC4 - Las situaciones problemáticas se resuelven con la aplicación del conocimiento organizacional. \\
\hline & $\begin{array}{l}\text { ApC5 - La estrategia de la organización se ajusta periódicamente tomando en cuenta el conoci- } \\
\text { miento organizacional. }\end{array}$ \\
\hline & ApC6 - El conocimiento organizacional se incorpora en los procesos de negocio de la organización. \\
\hline \multirow{5}{*}{ Transferencia } & TrC1 - Se crean y mantienen redes de interacción, formales e informales, para transferir conocimiento. \\
\hline & $\begin{array}{l}\text { TrC2 - Se despliegan herramientas y medios para apoyar la transferencia de conocimiento dentro } \\
\text { de las redes de interacción. }\end{array}$ \\
\hline & $\begin{array}{l}\text { TrC3 - Se construyen relaciones de confianza entre las personas de la organización, basadas en el } \\
\text { beneficio obtenido por transferir conocimiento. }\end{array}$ \\
\hline & $\begin{array}{l}\text { TrC4 - Se establecen mecanismos de realimentación para confirmar la efectividad en la transferencia } \\
\text { de conocimiento. }\end{array}$ \\
\hline & $\begin{array}{l}\text { TrC5 - Se construyen y mantienen diferentes tipos de espacios de interacción físicos o virtuales, que } \\
\text { permiten transferir conocimiento. }\end{array}$ \\
\hline \multirow{4}{*}{ Protección } & PrC1 - Se establecen incentivos para fomentar la protección del conocimiento de la organización. \\
\hline & PrC2 - Se usan tecnologías para proteger el conocimiento organizacional codificado. \\
\hline & PrC3 - Se identifican claramente el conocimiento protegido y el conocimiento abierto. \\
\hline & $\begin{array}{l}\text { PrC4 - Principios y prácticas de protección de conocimiento se incorporan dentro de los roles y las } \\
\text { responsabilidades de los miembros de la organización. }\end{array}$ \\
\hline \multirow{5}{*}{ Evaluación } & $\begin{array}{l}\text { EvC1 - } \\
\text { Se diseñan, adoptan y aplican métricas, mediciones o indicadores para el conocimiento } \\
\text { organizacional. }\end{array}$ \\
\hline & EvC2 - Se establecen metas de conocimiento \\
\hline & $\begin{array}{l}\text { EvC3 - El conocimiento organizacional se monitorea periódicamente utilizando las métricas, } \\
\text { mediciones o indicadores establecidos. }\end{array}$ \\
\hline & $\begin{array}{l}\text { EvC4 - El resultado del monitoreo del conocimiento organizacional es analizado y comparado con } \\
\text { metas de conocimiento establecidas, información histórica e información de otras organizaciones } \\
\text { (socios, competidores, proveedores, clientes). }\end{array}$ \\
\hline & amente reportes sobre el estado del conocimiento organizacional. \\
\hline
\end{tabular}

Fuente. Galvis-Lista y Sánchez-Torres, (2013)

Específicamente, se buscaron respuestas a las siguientes preguntas de investigación: ¿en las ODS de Colombia se reconoce la importancia de la GC? ¿Se han implementado estrategias, prácticas o herramientas de
GC? ¿Cuál es el estado de implementación de los procesos del MRPGC en términos de la obtención de sus resultados esperados? ¿En qué nivel se logran los propósitos de los procesos del MRPGC?, y ¿qué importancia tienen los procesos del MRPGC para las ODS?. 


\section{Método}

E I método utilizado fue el de encuesta, el cual se aplica en la investigación en ingeniería de software porque permite obtener datos para describir las reacciones de una población a un determinado método, herramienta o técnica, o para determinar tendencias y relaciones (Sjoberg, Dyba, \& Jorgensen, 2007). Es decir, se toma una imagen del estado actual de la implementación de alguna técnica o herramienta en un contexto particular de observación (Conradi \& Wang, 2003). Además, es posible identificar acuerdos existentes sobre la importancia, pertinencia y aplicabilidad de los elementos en estudio. Los trabajos (Cater-Steel, Toleman, \& Rout, 2005; Chow \& Cao, 2008; Dyba, 2005; Jiang, Klein, Hwang, Huang, \& Hung, 2004; Kannabiran \& Sankaran, 2011; Laanti, Salo, \& Abrahamsson, 2011; Niazi, Babar, \& Verner, 2010; Salo \& Abrahamsson, 2008; Vijayasarathy \& Turk, 2012) ilustran la aplicación de este método en investigaciones sobre procesos de software y los trabajos (Neves, Da Silva, Salomon, \& Santos, 2013; Reich, Gemino, \& Sauer, 2012; Ryan \& O'Connor, 2013; Singh, Singh, \& Sharma, 2014) muestran su uso en investigaciones sobre GC en ODS.

La recolección de datos se realizó utilizando un cuestionario en línea compuesto por 11 secciones, el cual fue diseñado para obtener respuestas que pudieran ser utilizadas en análisis descriptivos. En la primera sección se formularon preguntas para caracterizar a la persona que lo respondía y a la ODS a la cual se encontraba vinculada. En las siguientes ocho secciones se formularon preguntas relacionadas con la implementación de cada uno de los procesos descritos en el MRPGC, se tuvo una sección por cada proceso del MRPGC. También se dispuso una sección con preguntas sobre la importancia de los procesos de GC y por último una sección de síntesis y comentarios finales. El cuestionario fue revisado por expertos y validado con una prueba piloto que permitió hacer ajustes al cuestionario y se configuró en un servidor con la aplicación web LimeSurvey (Schmitz, 2013).

El análisis cuantitativo de los datos recolectados, se realizó utilizando métodos de estadística descriptiva como tablas de frecuencia, tablas de contingencia y medidas de tendencia central; y así identificar niveles de acuerdo o de consenso entre los participantes. En este sentido, para realizar el análisis de los resultados, se asumió el criterio utilizado por la ISO en la elaboración de estándares internacionales, quien define el consenso como la coincidencia de opiniones entre las dos terceras partes de los participantes $(66,7 \%)$ o más, y la mayoría cuando más de la mitad de los participantes coinciden (ISO/IEC, 2014). Además, los datos de las respuestas sobre el logro de los resultados de los procesos del MRPGC fueron analizados para determinar su consistencia interna con el coeficiente Alfa de Cronbach.

Con relación a la población objetivo del estudio, como se mencionó antes, estaba constituida por las ODS de Colombia. Particularmente, esta población estaba conformada, según datos del estudio realizado por FedeSoft a finales del año 2012 (Fedesoft, 2012), por 765 empresas. Para llegar a ese número, FedeSoft identificó que en Colombia existían 1813 empresas del sector TIC oficialmente registradas en las cámaras de comercio de cada ciudad. Sin embargo, encontraron que solamente 765 empresas tenían como objeto de negocio el desarrollo de software y otras 
actividades como consultoría o servicios de software (Fedesoft, 2012). Como la información de las empresas identificadas en el estudio de FedeSoft no estaba disponible para ser consultada libremente fue necesario identificar a las ODS a encuestar, utilizando datos disponibles libremente en Internet.

La identificación de las ODS se realizó consultando los portales web de FedeSoft (Fedesoft, 2014), ParqueSoft (Fundación PARQUESOFT, 2014), ESI Center (actualmente Fundación Tecnalia Colombia) (ESI Center SINERTIC Andino, 2015), y la Guía de Soluciones TIC de la empresa Datamarket Solutions SAS (Datamarket Solutions SAS, 2014). Posteriormente se buscaron los datos de contacto de personas con cargos directivos en los sitios web de las ODS identificadas y en varios motores de búsqueda de uso general. Para los casos en los que no se encontró el dato, se registró la dirección de contacto de información corporativa o la de servicio al cliente. Esta identificación se realizó en los meses de febrero y marzo de 2013, dando como resultado un listado de contactos de 475 ODS.

Los datos fueron cargados en la aplicación LimeSurvey (Schmitz, 2013) y se procedió a enviar los mensajes de correo electrónico con las invitaciones personalizadas para diligenciar el cuestionario. Estas últimas se enviaron el 1 de abril de 2013 y luego se hicieron seis recordatorios, uno cada dos semanas. La última respuesta fue recibida el 27 de junio de 2013. Al final se obtuvieron datos de 169 ODS de las 475 que fueron contactadas, obteniéndose una tasa de respuesta del $35,6 \%$. Así mismo, en relación con el número de empresas identificadas por FedeSoft (765 empresas), la tasa de respuesta fue del $22,1 \%$. Sumado a esto, las 169 ODS participantes son una muestra representativa del total de empresas de software del país (765) con un nivel de confianza del $95 \%$ y un intervalo de confianza del $6,66 \%$.

A partir delas respuestas recibidas, se realizó una caracterización de las ODS participantes de acuerdo con su ubicación geográfica, tamaño, actividad principal, certificaciones externas y orientación metodológica de sus prácticas de desarrollo de software. Con relación a la ubicación geográfica, las ODS participantes estaban ubicadas en 12 ciudades del país y la mayoría en las ciudades de Bogotá, Cali y Medellín. Así mismo, en cuanto al tamaño, la mayoría de ODS participantes se ubicó en la categoría de micro o pequeñas empresas según lo establecido en el artículo 43 de la Ley 1450 de 2011 (República de Colombia, 2011), sobre el tamaño empresarial y el número de empleados. Sobre la actividad económica principal, todas las ODS participantes declararon desarrollar actividades de ingeniería de software, tales como desarrollo, integración, consultoría, outsourcing, mantenimiento y soporte. Aquí, se destaca que la mayoría declaró como su actividad principal, el desarrollo de software. En cuanto a la certificación de los procesos se encontró que el $56,8 \%$ de las ODS participantes manifestó tener algún tipo de certificación. Las certificaciones referidas fueron ISO 9001, CMMI, IT Mark y MoProSoft. Además, el $62,1 \%$ manifestó tener una orientación hacia las prácticas ágiles de desarrollo de software y el restante $37,9 \%$ hacia las prácticas tradicionales. Estas distribuciones son coherentes con los datos presentados en el estudio de FedeSoft (Fedesoft, 2012, pp. 47-50, 64). A continuación se presentan los datos de caracterización básica de las ODS participantes (Tabla 3). 
Tabla 3. Caracterización básica de las ODS participantes

\begin{tabular}{|c|c|c|c|c|c|c|c|}
\hline Criterio & Categoría & Frecuencia & $\%$ & Criterio & Categoría & Frecuencia & $\%$ \\
\hline \multirow{13}{*}{ Ciudad } & Bogotá & 96 & 56,8 & \multirow{4}{*}{ Tamaño } & Micro (10 o menos) & 58 & 34,3 \\
\hline & Cali & 30 & 17,8 & & Pequeña (de 11 hasta 50) & 56 & 33,1 \\
\hline & Medellín & 20 & 11,8 & & Mediana (de 51 a 200) & 30 & 17,8 \\
\hline & Bucaramanga & 6 & 3,6 & & Grande (más de 200) & 25 & 14,8 \\
\hline & Manizales & 4 & 2,4 & \multirow{5}{*}{$\begin{array}{l}\text { Actividad } \\
\text { principal }\end{array}$} & Desarrollo de software & 134 & 79,3 \\
\hline & Pasto & 3 & 1,8 & & Integración de sistemas & 12 & 7,1 \\
\hline & Armenia & 2 & 1,2 & & $\begin{array}{l}\text { Consultoría en } \\
\text { aplicaciones }\end{array}$ & 10 & 5,9 \\
\hline & Barranquilla & 2 & 1,2 & & $\begin{array}{l}\text { Outsourcing de } \\
\text { aplicaciones }\end{array}$ & 7 & 4,1 \\
\hline & Cartagena & 2 & 1,2 & & $\begin{array}{l}\text { Servicio de soporte y } \\
\text { mantenimiento }\end{array}$ & 6 & 3,6 \\
\hline & Pereira & 2 & 1,2 & \multirow{4}{*}{ Certificación } & ISO 9001 & 72 & 42,6 \\
\hline & Ibagué & 1 & 0,6 & & CMMI & 35 & 20,7 \\
\hline & Popayán & 1 & 0,6 & & IT Mark & 12 & 7,1 \\
\hline & Total & 169 & 100,0 & & MoProSoft & 3 & 1,8 \\
\hline
\end{tabular}

Fuente. Elaboración propia de los autores, a partir de los datos recolectados con la encuesta.

\section{Resultados}

\subsection{Importancia e implementación de aspectos relacionados con GC}

En las respuestas a las primeras dos preguntas de investigación, se observó que el 78,7\% de los participantes manifestó que en su ODS se reconocía la importancia de la GC. Así mismo, el 55,6\% manifestó que en su ODS se había implementado algún aspecto relacionado con la GC.

\subsection{Implementación de los procesos de GC}

Con relación al estado de la implementación de los procesos, se obtuvieron los datos que se muestran más adelante (Tabla 4). En este punto, se aclara que la implementación de los procesos se evalúo a partir de las valoraciones sobre el logro de los resultados esperados de los procesos definidos en el MRPGC (GalvisLista y Sanchez-Torres, 2013). En solamente uno de los resultados esperados de uno de los procesos, el EvC5 «Se publican periódicamente reportes sobre el estado del conocimiento organizacional», se obtuvo un porcentaje superior a 50 en alguna de las categorías de respuesta. En ese caso, el 56,8\% manifestó que el resultado EvC5 «No se logra». Para el resto se obtuvieron proporciones diferentes, en donde los porcentajes más altos se ubicaron en las categorías «No se logra» y se logra «Aceptablemente».

Así mismo, se presenta un resumen de la tendencia central identificada (Tabla 5), mostrando, para cada proceso, el número 


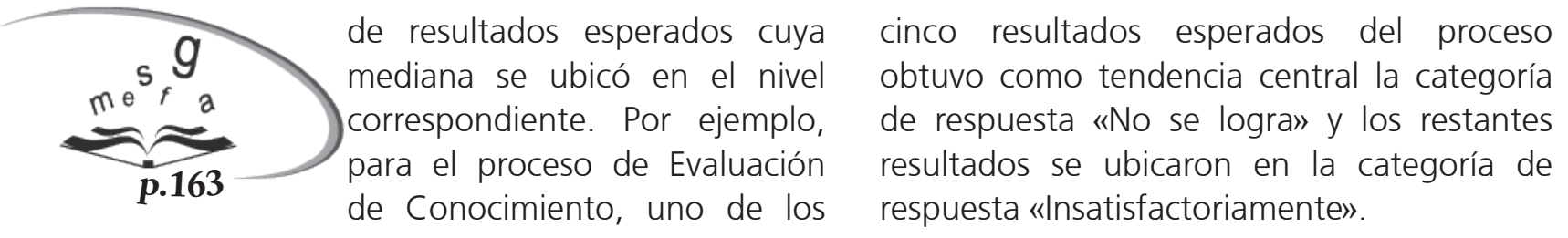

Tabla 4. Logro de los resultados esperados de los procesos

\begin{tabular}{|c|c|c|c|c|c|c|c|}
\hline Proceso & N & Resultado & $\begin{array}{c}\text { No se } \\
\text { logra } \\
\%\end{array}$ & $\begin{array}{c}\text { Insatisfacto- } \\
\text { riamente } \\
\%\end{array}$ & $\begin{array}{c}\text { Aceptablemente } \\
\%\end{array}$ & $\begin{array}{l}\text { En alto } \\
\text { grado \% }\end{array}$ & $\begin{array}{c}\text { Plenamente } \\
\%\end{array}$ \\
\hline \multirow{4}{*}{ Identificación } & \multirow{4}{*}{169} & $\mathrm{IdC} 1$ & 32,5 & 20,1 & 28,4 & 16,6 & 2,4 \\
\hline & & $\mathrm{IdC2}$ & 8,9 & 17,8 & 40,2 & 27,8 & 5,3 \\
\hline & & $\mathrm{IdC3}$ & 14,2 & 17,8 & 37,3 & 25,4 & 5,3 \\
\hline & & IdC4 & 18,3 & 23,1 & 33,1 & 22,5 & 3,0 \\
\hline \multirow{9}{*}{ Adquisición } & \multirow{9}{*}{159} & AdC1 & 14,5 & 23,9 & 38,4 & 21,4 & 1,9 \\
\hline & & $\mathrm{AdC2}$ & 18,2 & 13,8 & 39,6 & 22,0 & 6,3 \\
\hline & & AdC3 & 32,7 & 25,2 & 27,0 & 13,2 & 1,9 \\
\hline & & $\mathrm{AdC} 4$ & 21,4 & 28,3 & 22,0 & 21,4 & 6,9 \\
\hline & & $\mathrm{AdC5}$ & 10,7 & 22,6 & 27,7 & 30,2 & 8,8 \\
\hline & & AdC6 & 38,4 & 15,1 & 20,1 & 16,4 & 10,1 \\
\hline & & $\mathrm{AdC7}$ & 18,2 & 12,6 & 37,7 & 20,1 & 11,3 \\
\hline & & $\mathrm{AdC8}$ & 24,5 & 25,2 & 29,6 & 17,6 & 3,1 \\
\hline & & AdC9 & 10,7 & 10,7 & 37,1 & 30,8 & 10,7 \\
\hline \multirow{7}{*}{ Creación } & \multirow{7}{*}{150} & $\mathrm{CrC1}$ & 11,3 & 22,0 & 37,3 & 22,0 & 7,3 \\
\hline & & $\mathrm{CrC2}$ & 9,3 & 18,7 & 34,7 & 28,7 & 8,7 \\
\hline & & $\mathrm{CrC3}$ & 13,3 & 24,0 & 34,0 & 19,3 & 9,3 \\
\hline & & $\mathrm{CrC4}$ & 14,0 & 21,3 & 28,7 & 26,0 & 10,0 \\
\hline & & $\mathrm{CrC5}$ & 18,0 & 26,0 & 32,7 & 19,3 & 4,0 \\
\hline & & $\mathrm{CrC} 6$ & 17,3 & 20,0 & 28,0 & 23,3 & 11,3 \\
\hline & & $\mathrm{CrC7}$ & 28,0 & 22,0 & 26,7 & 15,3 & 8,0 \\
\hline \multirow{7}{*}{ Codificación } & \multirow{7}{*}{143} & CoC1 & 20,3 & 23,1 & 31,5 & 21,7 & 3,5 \\
\hline & & $\mathrm{CoC2}$ & 28,7 & 28,0 & 23,1 & 18,9 & 1,4 \\
\hline & & $\mathrm{CoC} 3$ & 39,9 & 23,1 & 20,3 & 16,1 & 0,7 \\
\hline & & CoC4 & 30,1 & 25,9 & 21,7 & 17,5 & 4,9 \\
\hline & & CoC5 & 29,4 & 22,4 & 25,2 & 16,1 & 7,0 \\
\hline & & CoC6 & 42,7 & 20,3 & 23,8 & 11,2 & 2,1 \\
\hline & & $\mathrm{CoC7}$ & 31,5 & 23,1 & 27,3 & 15,4 & 2,8 \\
\hline \multirow{6}{*}{ Aplicación } & \multirow{6}{*}{137} & $\mathrm{ApC1}$ & 15,3 & 19,7 & 35,8 & 21,2 & 8,0 \\
\hline & & $\mathrm{ApC2}$ & 10,9 & 13,9 & 34,3 & 29,9 & 10,9 \\
\hline & & $\mathrm{ApC} 3$ & 8,8 & 12,4 & 38,0 & 30,7 & 10,2 \\
\hline & & $\mathrm{ApC} 4$ & 8,8 & 14,6 & 39,4 & 26,3 & 10,9 \\
\hline & & ApC5 & 15,3 & 15,3 & 38,7 & 23,4 & 7,3 \\
\hline & & ApC6 & 13,9 & 14,6 & 35,0 & 28,5 & 8,0 \\
\hline
\end{tabular}


Tabla 4. Logro de los resultados esperados de los procesos (Continuación)

\begin{tabular}{|c|c|c|c|c|c|c|c|}
\hline Proceso & $\mathbf{N}$ & Resultado & $\begin{array}{c}\text { No se } \\
\text { logra } \\
\%\end{array}$ & $\begin{array}{c}\text { Insatisfacto- } \\
\text { riamente } \\
\%\end{array}$ & $\begin{array}{c}\text { Aceptable- } \\
\text { mente } \\
\%\end{array}$ & $\begin{array}{c}\text { En alto } \\
\text { grado \% }\end{array}$ & $\begin{array}{c}\text { Plenamente } \\
\%\end{array}$ \\
\hline \multirow{5}{*}{ Transferencia } & \multirow{5}{*}{133} & $\operatorname{TrC} 1$ & 12,8 & 23,3 & 31,6 & 26,3 & 6,0 \\
\hline & & $\operatorname{TrC2}$ & 15,0 & 24,8 & 34,6 & 19,5 & 6,0 \\
\hline & & $\operatorname{TrC} 3$ & 6,0 & 17,3 & 30,1 & 34,6 & 12,0 \\
\hline & & $\operatorname{TrC} 4$ & 21,1 & 25,6 & 24,8 & 24,1 & 4,5 \\
\hline & & $\operatorname{TrC} 5$ & 9,0 & 24,1 & 32,3 & 25,6 & 9,0 \\
\hline \multirow{4}{*}{ Protección } & \multirow{4}{*}{132} & $\operatorname{PrC} 1$ & 47,0 & 22,7 & 17,4 & 7,6 & 5,3 \\
\hline & & $\operatorname{PrC2}$ & 20,5 & 22,0 & 28,0 & 20,5 & 9,1 \\
\hline & & $\operatorname{PrC} 3$ & 24,2 & 20,5 & 22,0 & 22,0 & 11,4 \\
\hline & & $\operatorname{PrC} 4$ & 20,5 & 16,7 & 30,3 & 23,5 & 9,1 \\
\hline \multirow{5}{*}{ Evaluación } & \multirow{5}{*}{132} & EvC1 & 37,1 & 28,8 & 18,9 & 12,9 & 2,3 \\
\hline & & $\mathrm{EvC2}$ & 43,2 & 26,5 & 18,2 & 9,8 & 2,3 \\
\hline & & EvC3 & 46,2 & 23,5 & 17,4 & 10,6 & 2,3 \\
\hline & & EvC4 & 41,7 & 18,9 & 20,5 & 15,2 & 3,8 \\
\hline & & EvC5 & 56,8 & 15,2 & 19,7 & 7,6 & 0,8 \\
\hline
\end{tabular}

* Las celdas sombreadas resaltan las categorías en donde se ubicó la mediana.

Fuente. Elaboración propia de los autores, a partir de los datos recolectados con la encuesta.

Tabla 5. Resumen de la identificación de la tendencia central en el logro de los resultados esperados

\begin{tabular}{|l|c|c|c|c|c|c|}
\hline \multicolumn{1}{|c|}{ Proceso } & Resultados & No se logra & $\begin{array}{c}\text { Insatisfacto- } \\
\text { riamente }\end{array}$ & $\begin{array}{c}\text { Aceptable- } \\
\text { mente }\end{array}$ & $\begin{array}{c}\text { En alto } \\
\text { grado }\end{array}$ & Plenamente \\
\hline Identificación & 4 & - & 1 & 3 & - & - \\
\hline Adquisición & 9 & - & 3 & 5 & 1 & - \\
\hline Creación & 7 & - & 1 & 6 & - & - \\
\hline Codificación & 7 & - & 6 & 1 & - & - \\
\hline Aplicación & 6 & - & - & 6 & - & - \\
\hline Transferencia & 5 & - & - & 5 & - & - \\
\hline Protección & 4 & - & 1 & 3 & - & - \\
\hline Evaluación & 5 & 1 & 4 & - & - & - \\
\hline
\end{tabular}

Fuente. Elaboración propia de los autores, a partir de los datos recolectados con la encuesta.

Tomando los datos obtenidos sobre el logro de los resultados esperados de los procesos, se calculó la consistencia interna con el coeficiente Alfa de Cronbach. Se puede observar (Tabla 6) que en todos los procesos, el valor del coeficiente fue superior a 0,88. También se destaca que el proceso con el valor más alto del coeficiente fue el de Codificación de Conocimiento con 0,965. 
Tabla 6. Coeficiente Alfa de Cronbach para cada proceso

\begin{tabular}{|l|l|l|l|}
\hline \multicolumn{1}{|c|}{ Proceso } & \multicolumn{1}{c|}{$\begin{array}{c}\text { N } \\
\text { válido }\end{array}$} & \multicolumn{1}{c|}{ Alfa de } & Ítems \\
\hline Cronbach & & & \\
\hline Identificación & 169 & 0,904 & 4 \\
\hline Adquisición & 159 & 0,880 & 9 \\
\hline Creación & 150 & 0,916 & 7 \\
\hline Codificación & 143 & 0,965 & 7 \\
\hline Aplicación & 137 & 0,942 & 6 \\
\hline Transferencia & 133 & 0,924 & 5 \\
\hline Protección & 132 & 0,893 & 4 \\
\hline Evaluación & 132 & 0,957 & 5 \\
\hline
\end{tabular}

Fuente. Elaboración propia propia de los autores, a partir de los datos recolectados con la encuesta.

\subsection{Logro de los propósitos de los procesos de GC}

Sobre el logro de los propósitos de los procesos, estos se presentan a continuación (Tabla 7). Allí se observa, que para todos los procesos, el porcentaje más alto se muestra en la categoría "Aceptablemente», ubicada en la mitad de la escala. Además, se encontró que el proceso de Evaluación de Conocimiento fue el que obtuvo el porcentaje más alto en la categoría «No se logra».

Tabla 7. Logro de los propósitos de los procesos

\begin{tabular}{|l|c|c|c|c|c|c|}
\hline \multicolumn{1}{|c|}{ Proceso } & $\begin{array}{c}\mathbf{N} \\
\text { válido }\end{array}$ & $\begin{array}{c}\text { No se logra } \\
\%\end{array}$ & $\begin{array}{c}\text { Insatisfacto- } \\
\text { riamente } \\
\%\end{array}$ & $\begin{array}{c}\text { Aceptable- } \\
\text { mente } \\
\%\end{array}$ & $\begin{array}{c}\text { En alto } \\
\text { grado } \\
\%\end{array}$ & $\begin{array}{c}\text { Plenamente } \\
\%\end{array}$ \\
\hline Identificación & 169 & 16,6 & 19,5 & 50,9 & 11,8 & 1,2 \\
\hline Adquisición & 159 & 11,9 & 20,8 & 46,5 & 19,5 & 1,3 \\
\hline Creación & 151 & 9,9 & 24,5 & 41,7 & 20,5 & 3,3 \\
\hline Codificación & 143 & 23,8 & 29,4 & 31,5 & 14,7 & 0,7 \\
\hline Aplicación & 136 & 12,5 & 17,6 & 44,9 & 22,8 & 2,2 \\
\hline Transferencia & 133 & 9,0 & 23,3 & 40,6 & 24,8 & 2,3 \\
\hline Protección & 132 & 22,0 & 15,2 & 39,4 & 18,2 & 5,3 \\
\hline Evaluación & 132 & 31,1 & 18,9 & 34,8 & 14,4 & 0,8 \\
\hline
\end{tabular}

Fuente. Elaboración propia con base en los datos recolectados con la encuesta.

\subsection{Importancia de los procesos de GC}

Con relación a la importancia de los procesos, se obtuvieron los resultados que se presentan más adelante (Tabla 8). Allí se observa que, todos los procesos, exceptuando el de Evaluación de Conocimiento, fueron valorados mayoritariamente en la categoría
«Muy Importante» (Tabla 8). Ahora bien, si se suman los porcentajes de las dos categorías que denotan mayor importancia, es decir «lmportante»y «Muy importante», se obtienen valores superiores a $88,7 \%$, lo cual significa la existencia de consenso en que para las ODS todos los procesos del MRPGC son, al menos, importantes. 
Tabla 8. Importancia de los procesos para las ODS

\begin{tabular}{|l|c|c|c|c|c|c|}
\hline \multicolumn{1}{|c|}{ Proceso } & $\begin{array}{c}\mathbf{N} \\
\text { válido }\end{array}$ & $\begin{array}{c}\text { Nada } \\
\text { importante } \\
\%\end{array}$ & $\begin{array}{c}\text { Poco } \\
\text { importante } \\
\%\end{array}$ & $\begin{array}{c}\text { Moderadamente } \\
\text { Importante } \\
\%\end{array}$ & $\begin{array}{c}\text { Importante } \\
\%\end{array}$ & $\begin{array}{c}\text { Muy } \\
\text { Importante } \\
\%\end{array}$ \\
\hline Identificación & 169 & - & 1,8 & 5,3 & 32,5 & 60,4 \\
\hline Adquisición & 159 & 1,3 & 0,6 & 6,9 & 34,6 & 56,6 \\
\hline Creación & 150 & - & - & 6,0 & 32,0 & 62,0 \\
\hline Codificación & 143 & - & 1,4 & 8,4 & 35,7 & 54,5 \\
\hline Aplicación & 137 & - & - & 5,1 & 32,1 & 62,8 \\
\hline Transferencia & 133 & - & - & 5,3 & 36,1 & 58,6 \\
\hline Protección & 132 & - & 0,8 & 6,1 & 30,3 & 62,9 \\
\hline Evaluación & 132 & - & 2,3 & 9,1 & 45,5 & 43,2 \\
\hline
\end{tabular}

Fuente. Elaboración propia de los autores, a partir de los datos recolectados con la encuesta.

\section{Discusión}

T as respuestas, en su mayoría positivas, sobre el reconocimiento de la importancia de la GC y la implementación de aspectos relacionados con la GC en las ODS participantes, evidencian la importancia de este tema en el sector. También significan la existencia de un ambiente propicio para la adopción de modelos de referencia como el MRPGC, así como, de otros elementos metodológicos relacionados con la GC. Sin embargo, el hecho de que más del $20 \%$ de los participantes manifestó que en su ODS no se reconocía la importancia de la GC se considera un aspecto negativo si se toma en consideración que el objeto de negocio y el principal recurso de las ODS es el conocimiento.

Las valoraciones del logro de los resultados esperados y de los propósitos de los procesos del MPRGC, también sustentan, de manera general, la idea de un estado de implementación apenas aceptable. Sin embargo, las valoraciones del logro de los resultados del proceso de codificación de conocimiento llaman la atención, pues los porcentajes más altos en seis de los siete resultados esperados del proceso se dieron en la categoría «No se logra». Así mismo, al tomar en consideración los porcentajes de las dos categorías más bajas de la escala, se observa que en la mayoría de las ODS participantes, la implementación de este proceso no alcanza a ser ni siquiera aceptable. La discusión sobre este proceso en particular es clave porque según la literatura consultada, este es el proceso que más se ha trabajo en el contexto de las ODS. Además, en muchas organizaciones, los primeros pasos que se dan en el camino de la GC están asociados directamente con la codificación del conocimiento de las personas, por consiguiente, se esperaba un estado más maduro de implementación de este proceso.

En esta misma línea, llaman la atención las valoraciones de la implementación del proceso de Evaluación de Conocimiento, en donde los porcentajes en la categoría «No se logra» son mucho mayores con relación al proceso de codificación de conocimiento. No evaluar el conocimiento de forma sistemática y no definir metas de desarrollo del conocimiento organizacional, es un aspecto crítico que podría considerarse parte de las razones por las cuales se da el bajo grado de especialización, la preferencia por desarrollar líneas de negocio tradicionales en lugar de 
aprovechar oportunidades identificadas en las tendencias del mercado de software a nivel mundial, y la poca o nula dedicación a la investigación, desarrollo e innovación (Fedesoft, 2012). Además, sin evaluación de conocimiento es probable que cualquier iniciativa relacionada con la GC no prospere en el tiempo, porque no habría forma de determinar sus efectos en la organización y tampoco se tendrían elementos de juicio para establecer si valió la pena la inversión de recursos financieros, humanos y tecnológicos.

Por otra parte, con la evaluación del estado de implementación de los procesos de GC se evidencia la existencia de un escenario favorable para el trabajo con el MRPGC en la industria de software de Colombia, bien sea utilizándolo como referencia para la evaluación de los procesos o para la identificación de aspectos a mejorar. También está el hecho de tener porcentajes mayores que cero en las dos categorías superiores de la escala, lo cual indica que los resultados de los procesos descritos en el MRPGC son alcanzables por las ODS de Colombia y que todos los elementos contemplados en esta versión del MRPGC son pertinentes para este sector. Sumado a esto, los valores obtenidos para el coeficiente Alfa de Cronbach, indican la consistencia interna de los elementos valorados, es decir, de los conjuntos de resultados de cada proceso del MRPGC. El argumento de la pertinencia de los planteamientos del MRPGC también se soporta en las valoraciones de la importancia de los procesos para las ODS, en dónde se dieron consensos en las valoraciones de los ocho procesos como «Importantes» o "Muy importantes».

\section{Conclusiones}

L os resultados y la discusión presentados, dieron respuesta a las preguntas de interés que motivaron la ejecución de esta investigación. En síntesis, se encontró que en la mayoría de ODS participantes se reconoce la importancia de los procesos de GC, y en una gran proporción de ODS, se han implementado aspectos relacionados con la GC. Sin embargo, se encontró que la implementación de los procesos de GC en las ODS en estudio, observada desde la perspectiva de la obtención de sus resultados esperados y del logro de sus propósitos, tiene un estado apenas aceptable.

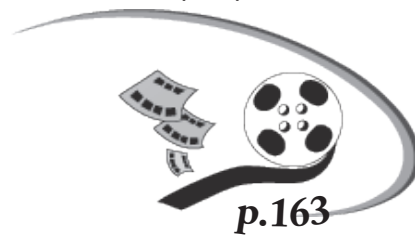

Esto evidencia la necesidad de fortalecer estos procesos, para que las organizaciones de la industria del software de Colombia puedan generar ventajas competitivas sostenibles y superar aspectos negativos, como el bajo grado de especialización, la dedicación casi exclusiva a líneas de negocio tradicionales, y la poca o nula dedicación a actividades de investigación, desarrollo e innovación.

Esta conclusión se refuerza al tomar en consideración los resultados obtenidos con la valoración de la importancia de los procesos de GC descritos en el MRPGC, pues la mayoría de ODS participantes manifestó que los ocho procesos son de muy alta importancia. Así mismo, se evidenció la pertinencia de utilizar el MRPGC como guía de referencia para evaluar el estado de implementación de los procesos de GC en las ODS de Colombia, permitiendo que cada organización participante se evaluara con un marco de referencia que agrupa tanto propósitos como resultados esperados. Se observó que el MRPGC puede constituirse como un modelo de referencia que permite la identificación de los aspectos a mejorar para que las ODS logren avances 
en la implementación de los procesos de GC. Particularmente, las ODS participantes hicieron observaciones cualitativas en las que expresaron que el MRPGC les permitió tener claridad sobre los propósitos que los procesos de GC deberían alcanzar, así como los resultados esperados de la implementación exitosa de tales procesos.

En relación con el diseño metodológico utilizado, se evidenció la viabilidad de realizar este tipo de estudios en la industria de software de Colombia, en gran medida gracias al uso de herramientas de recolección de datos en línea. Sin embargo, esto también constituye la más importante limitación del trabajo realizado, por lo cual se propone como trabajo futuro la indagación sobre el estado de implementación de los procesos de GC utilizando otros métodos de investigación que permitan mayor interacción con las organizaciones, tales como el estudio de caso o la investigación-acción. Así mismo, se sugiere la realización de estudios que indaguen sobre la relación entre las características particulares de las organizaciones -tamaño, edad, ubicación geográfica, enfoque metodológico, entre otros- y el estado de implementación de proceso de GC, aplicando métodos estadísticos de análisis de asociación y correlación entre variables, y métodos multivariados. Por último, se propone la idea de replicar este estudio con ODS de otros países de América Latina, con el fin de hacer una comparación e identificar las ODS con mayor avance en la implementación de procesos de GC.

\section{Referencias bibliográficas}

IBjørnson, F. O., \& Dingsøyr, T. (2008). Knowledge management in software engineering: A systematic review of studied concepts, findings and research methods used. Information and Software Technology, 50(11), 1055-1068. Recuperado de: http:// doi.org/10.1016/j.infsof.

Cater-Steel, A., Toleman, M., \& Rout, T. (2005). Addressing the challenges of replications of surveys in software engineering research (p. 10). Presented at the Empirical Software Engineering, 2005. 2005 International Symposium on.

Chow, T., \& Cao, D.-B. (2008). A survey study of critical success factors in agile software projects. Journal of Systems and Software, 81(6), 961-971. Recuperado de: http:// doi.org/10.1016/j.jss.

CIDEI, CINTEL, ESI Center SINERTIC Andino, Fundación TECNALIA, IKEI, \& Research \&
Consultancy. (2013). Visión estratégica del sector de Software y servicios asociados. Bogotá, Colombia: Ministerio de Tecnologías de la Información y las Comunicaciones. Recuperado de: http://www.fiti.gov.co/Images/Recursos/ resumenejecutivoves-v130827.pdf

Conradi, R., \& Wang, A. I. (2003). Empirical Methods and Studies in Software Engineering: Experiences from ESERNET (1st ed.). Springer.

Datamarket Solutions SAS. (2014). Proveedores y Empresas de Software en Colombia -Guía Soluciones TIC. Recuperado de: http://www.guiadesolucionestic.com/ component/sobi2/?catid=3

Departamento Nacional de Planeación. (2007). Agenda Interna para la Productividad y Competitividad -Documento Sectorial Software. 
Dingsøyr, T., Bjornson, F. O., \& Shull, F. (2009). What Do We Know about Knowledge Management? Practical Implications for Software Engineering. Software, IEEE, 26(3), 100-103.

Dyba, T. (2005). An empirical investigation of the key factors for success in software process improvement. IEEE Transactions on Software Engineering, 31(5), 410-424. Recuperado de: http://doi.org/10.1109/ TSE.2005.53

ESI Center SINERTIC Andino. (2015). ES/Center Sinertic. Retrieved January 30, 2015, from. Recuperado de: http://www.esicentersinertic.org/index.php/quienes-somos

Fedesoft. (2011). Sector de TI en colombia año 2010 y proyecciones 2013 (Reporte de Estadísticas). Bogotá, Colombia: Federación Colombiana de la Industria del Software. Recuperado de: http:// www.fedesoft.org/sites/default/files/TI_ Colombia_Cifras_Fedesoft.pdf

Fedesoft. (2012). Estudio de la caracterización de productos y servicios de la industria de software y servicios asociados 2012. Bogotá, Colombia': Federación Colombiana de la Industria del Software. Recuperado de: http://www.fedesoft.org/Downloads/ EstudiocifrassectorSW2012.pdf

Fedesoft. (2014). Catálogo de Empresas Fedesoft I Fedesoft I Federación Colombiana de la Industria de Software y TI. Recuperado de: http://fedesoft.org/ catalogo-de-empresas/

Fundación PARQUESOFT. (2014). Empresas -PARQUESOFT. Recuperado de: http:// parquesoft.com/index. php?option=com _ content\&view=category\&layout= blog\&id=31\&ltemid=139
Galvis-Lista, E., \& Sanchez-Torres, J. M. (2013). ModelodereferenciadeprocesosdeGestión de Conocimiento para Organizaciones Desarrolladoras de Software de Colombia 1. Recuperado de: http://dx.doi. org/10.13140/2.1.4404.0960

GRUPO DE ESTUDIOS SECTORIALES-DNP. (2008). Industria del Software en Colombia. Bogotá, Colombia: Departamento Nacional de Planeación. Recuperado de: http://www.fedesoft. org/sites/default/files/DNP_Cadena_de_ software_y_servicios_asociados_0.pdf

ISO/IEC. (2014). ISO/IEC Directives, Part 1: Procedures for the technical work. 2014 (11th ed.). Ginebra, Suiza: ISO/IEC.

Jiang, J. J., Klein, G., Hwang, H.-G., Huang, J., \& Hung, S.-Y. (2004). An exploration of the relationship between software development process maturity and project performance. Information and Management, 41(3), 279-288. Recuperado de: http://doi.org/10.1016/S03787206(03)00052-1

Kannabiran, G., \& Sankaran, K. (2011). Determinants of software quality in offshore development - An empirical study of an Indian vendor. Information and Software Technology, 53(11), 1199-1208. Recuperado de: http://doi.org/10.1016/j. infsof.2011.05.001

Laanti, M., Salo, O., \& Abrahamsson, P. (2011). Agile methods rapidly replacing traditional methods at Nokia: A survey of opinions on agile transformation. Information and Software Technology, 53(3), 276-290. http://doi.org/10.1016/j. infsof.2010.11.010 
McKinsey \& Company. (2008). Desarrollando el sector de Tl como uno de Clase Mundial. Bogotá, Colombia: Ministerio de Comercio, Industria y Turísmo. Recuperado de: http:// www.transformacionproductiva.gov.co/ descargar.php?id=40516

Ministerio de Comercio, Industria y Turísmo. (2008). Proyecto para desarrollar sectores emergentes de clase mundial - propuesta del sector software y servicios asociados. Ministerio de Comercio, Industria y Turísmo.

Ministerio de Comunicaciones. (2008). Plan Nacional de Tecnologías de la Información y las Comunicaciones.(s.c) (s.e).

Neves, S. M., Da Silva, C. E. S., Salomon, V. A. P., \& Santos, A. L. A. (2013). Knowledgebased risk management: Survey on Brazilian software development enterprises 206 AISC.

Niazi, M., Babar, M. A., \& Verner, J. M. (2010). Software Process Improvement barriers: A cross-cultural comparison. Information and Software Technology, 52(11), 1204-1216. Recuperado de: http://doi.org/10.1016/j. infsof.2010.06.005

Parra Castrillón, J. E. (2008). Factores Críficos de Éxito e Hipótesis sobre la Industria del Software en Colombia. Conisideraciones Contextuales y Académicas, 5.

Reich, B. H., Gemino, A., \& Sauer, C. (2012). Knowledge management and projectbased knowledge in it projects: A modeland preliminary empirical results. International Journal of Project Management, 30(6), 663-674. Recuperado de: http://doi. org/10.1016/j.jproman.2011.12.003
República de Colombia (2011). Ley 1450 de 2011 (2011).

Ryan, S., \& O'Connor, R. V. (2013). Acquiring and Sharing tacit knowledge in software development teams: An empirical study. Information and Software Technology, 55(9), 1614-1624. Recuperado de: http:// doi.org/10.1016/j.infsof.2013.02.013

Salo, O., \& Abrahamsson, P. (2008). Agile methods in European embedded software development organisations: A survey on the actual use and usefulness of Extreme Programming and Scrum. IET Software, 2(1), 58-64. Recuperado de: http://doi. org/10.1049/iet-sen:20070038

Schmitz, C. (2013). LimeSurvey - The Open Source Survey Application (Version 2.05). LimeSurvey. Recuperado de: https://www. limesurvey.org/es/

Singh, A., Singh, K., \& Sharma, N. (2014). Agile knowledge management: a survey of Indian perceptions. Innovations in Systems and Software Engineering, 10(4), 297-315. Recuperado de: http://doi. org/10.1007/s11334-014-0237-z

Sjoberg, D. I. K., Dyba, T., \& Jorgensen, M. (2007). The Future of Empirical Methods in Software Engineering Research (pp. 358-378). Presented at the Future of Software Engineering, 2007. FOSE '07.

Vijayasarathy, L., \& Turk, D. (2012). Drivers of agile software development use: Dialectic interplay between benefits and hindrances. Information and Software Technology, 54(2), 137-148. Recuperado de:http:// doi.org/10.1016/j.infsof.2011.08.003 
